\title{
Effect of IV alteplase on the ischemic brain lesion at 24-48 hours after ischemic stroke
}

Grant Mair, MD, Rüdiger von Kummer, MD, Zoe Morris, MBBS, Anders von Heijne, MD, Nick Bradey, MBBS, Lesley Cala, MD, André Peeters, MD, Andrew J. Farrall, MD, Alessandro Adami, MD, Gillian Potter, MD, Peter A.G. Sandercock, DM, Richard I. Lindley, MD, and Joanna M. Wardlaw, MD, for the IST-3 Collaborative Group

Neurology ${ }^{\circledR}$ 2018;91:e2067-e2077. doi:10.1212/WNL.0000000000006575

\section{Abstract}

\section{Objective}

To determine whether alteplase alters the development of ischemic lesions on brain imaging after stroke.

\section{Methods}

The Third International Stroke Trial (IST-3) was a randomized controlled trial of IV alteplase for ischemic stroke. We assessed CT or brain MRI at baseline (pretreatment) and 24 to 48 hours posttreatment for acute lesion visibility, extent, and swelling, masked to all other data. We analyzed associations between treatment allocation, change in brain tissue appearances between baseline and follow-up imaging, and 6-month functional outcome in IST-3. We performed a meta-analysis of randomized trials of alteplase vs control with pre- and postrandomization imaging.

\section{Results}

Of 3,035 patients recruited in IST-3, 2,916 had baseline and follow-up brain imaging. Progression in either lesion extent or swelling independently predicted poorer 6-month outcome (adjusted odds ratio $[\mathrm{OR}]=0.92,95 \%$ confidence interval $[\mathrm{CI}] 0.88-0.96, p<0.001$; OR = 0.73 , 95\% CI 0.66-0.79, $p<0.001$, respectively). Patients allocated alteplase were less likely than controls to develop increased lesion visibility at follow-up ( $\mathrm{OR}=0.77,95 \% \mathrm{CI} 0.67-0.89$, $p<0.001)$, but there was no evidence that alteplase reduced progression of lesion extent or swelling. In meta-analysis of 6 trials including IST-3 $(n=4,757)$, allocation to alteplase was associated with a reduction in ischemic lesion extent on follow-up imaging $(\mathrm{OR}=0.85,95 \% \mathrm{CI}$ $0.76-0.95, p=0.004)$.

\section{Conclusion}

Alteplase was associated with reduced short-term progression in lesion visibility. In metaanalysis, alteplase reduced lesion extent. These findings may indicate that alteplase improves functional outcome by reducing tissue damage.

\section{Classification of evidence}

This study provides Class II evidence that IV alteplase impedes the progression of ischemic brain lesions on imaging after stroke.

\section{Correspondence}

Prof. Wardlaw

joanna.wardlaw@ed.ac.uk

\section{MORE ONLINE}

$\rightarrow$ Class of Evidence

Criteria for rating therapeutic and diagnostic studies

NPub.org/coe

\footnotetext{
From Edinburgh Imaging, and UK Dementia Research Institute at the University of Edinburgh and Centre for Clinical Brain Sciences (G.M., Z.M., A.J.F., J.M.W.), and Division of Clinical Neurosciences (P.A.G.S.), University of Edinburgh, UK; Department of Neuroradiology (R.v.K.), Dresden University Stroke Centre, Germany; Danderyd Hospital (A.v.H.), Stockholm, Sweden; Neuroradiology (N.B.), James Cook University Hospital, Middlesborough, UK; School of Medicine (L.C.), University of Western Australia; Cliniques Universitaires St Luc (A.P.), Neurologie, Belgium; Stroke Center (A.A.), Department of Neurology, IRCCS Sacro Cuore Don Calabria Hospital, Negrar, Verona, Italy; Department of Neuroradiology (G.P.), Salford Royal NHS Foundation Trust, Manchester, UK; and Westmead Hospital Clinical School and The George Institute for Global Health (R.I.L.), University of Sydney, Australia.

Go to Neurology.org/N for full disclosures. Funding information and disclosures deemed relevant by the authors, if any, are provided at the end of the article. 


\section{Glossary}

ASPECTS = Alberta Stroke Program Early CT Score; CI = confidence interval; DWI = diffusion-weighted imaging; ECASS = European Cooperative Acute Stroke Study; IQR = interquartile range; IST-3 = Third International Stroke Trial; MCA = middle cerebral artery; NIHSS = NIH Stroke Scale; OR = odds ratio.

In patients presenting acutely with ischemic stroke, brain imaging can demonstrate ischemia and infarction based on water shifts within brain tissue. ${ }^{1}$ On CT, brain tissue becomes gradually more hypoattenuated as its water content increases. ${ }^{2}$ Within the first few hours after stroke onset, there is a reduction in gray matter attenuation so that gray matter becomes of similar attenuation to normal white matter resulting in the welldescribed loss of visibility of the insular ribbon, basal ganglia, or affected cortex. ${ }^{3,4}$ These early attenuation changes of the brain are often not apparent immediately after symptom onset, but by 24 hours, affected brain is usually obviously hypoattenuated compared with normal brain, ${ }^{5}$ and is specific for infarction. ${ }^{6} \mathrm{On}$ MRI, diffusion-weighted imaging (DWI) can show hyperintensity in ischemic tissue within minutes of stroke onset. Hours later, the lesion also becomes hyperintense on other T2weighted sequences ${ }^{7}$ and, similar to hypoattenuation on CT, this later change usually indicates infarction. ${ }^{8}$ Both CT and MRI show brain tissue swelling as a secondary indicator of injury.

Treatment with IV alteplase within the first few hours after stroke improves long-term clinical outcome and accelerates disappearance of arterial obstruction. ${ }^{9-11}$ However, it is unclear whether IV alteplase modifies progression of the appearance of acutely affected brain tissue on imaging (i.e., the ischemic lesion) over the first 24 to 48 hours after stroke, or whether any shortterm alteplase-related alteration in lesion progression might explain the long-term improvement in functional outcome.

The Third International Stroke Trial (IST-3) was a large multicenter, randomized controlled trial testing IV alteplase given within 6 hours of ischemic stroke. ${ }^{12}$ In the present analysis, our aim was to assess the effect of alteplase on any change in ischemic lesion appearance on CT or MRI between pretreatment (baseline) and 24- to 48-hour posttreatment follow-up imaging. We investigated whether alteplase modified short-term progression of CT hypoattenuation or magnetic resonance T2-weighted hyperintensity (lesion visibility), lesion extent, or tissue swelling compared to control and tested whether alteration in short-term progression of the lesion appearance predicted long-term functional outcome after ischemic stroke. We set our results in the context of a meta-analysis of all available data from randomized controlled trials.

\section{Methods}

\section{Standard protocol approvals, registrations, and patient consents}

IST-3 was an international, multicenter PROBE (prospective, randomized, open-label, blinded endpoint) trial of
IV alteplase (recombinant tissue plasminogen activator) for ischemic stroke. ${ }^{12}$ Ethical approval was granted by the Scotland A research ethics committee and by local ethics committees. IST-3 was registered with Current Controlled Trials (ISRCTN25765518). Enrollment, data collection, and CONSORT (Consolidated Standards of Reporting Trials) compliance have been described. ${ }^{12,13}$

Briefly, adult patients with acute stroke of any severity (assessed with the NIH Stroke Scale [NIHSS]), with no upper age limit, were eligible if treatment could be started within 6 hours of symptom onset and brain imaging had excluded intracranial hemorrhage and structural stroke mimics. Informed consent for research was obtained for all patients. The full trial protocol is available: dcn.ed.ac.uk/ist3/. IST-3 primary results are published. ${ }^{12}$

Patients were randomly allocated to IV alteplase $(0.9 \mathrm{mg} / \mathrm{kg})$ or control. Treating clinicians used an automated telephone or online system to enter baseline data and obtain a randomized treatment allocation. Excepting the first 276 patients (doubleblind phase), treatment was given open label. Patients were followed up at 6 months by postal or telephone questionnaire to assess functional status with the Oxford Handicap Scale ${ }^{14}$ by assessors who were masked to clinical and imaging findings and treatment allocation.

\section{Brain imaging}

The IST-3 imaging protocol has been described ${ }^{4,12,15}$ : CT or MRI was required pretreatment at baseline and followup. For CT, the maximum slice thickness was $5 \mathrm{~mm}$ through the posterior fossa and $10 \mathrm{~mm}$ for the cerebrum, but most CT was performed with thinner slices. MRI included T1, $\mathrm{T} 2$, DWI, fluid-attenuated inversion recovery, and $\mathrm{T} 2{ }^{*}$. DICOM (Digital Imaging and Communications in Medicine) was collated centrally and anonymized. We excluded patients from the present analysis if images were not received centrally.

\section{Image analysis}

A panel of 10 experienced neuroradiologists and neurologists assessed imaging with a secure online viewing tool, which was developed after extensive review of early ischemic signs ${ }^{3}$ and observer reliability testing., ${ }^{46,17}$ The Systematic Image Review System includes a validated data collection pro forma (available at ed.ac.uk/edinburgh-imaging/image-analysistools), which underwent extensive observer reliability testing $^{16,17}$ to ensure there was satisfactory agreement ( $\kappa$ $>0.7$ ) between readers for all imaging features assessed. ${ }^{4}$ Image assessors were masked to clinical data and treatment 
allocation. We scored baseline imaging (prerandomization) separately and blindly to follow-up imaging (24-48 hours after stroke onset) and vice versa, although assessors were aware if imaging was acquired at baseline or follow-up.

We reviewed all baseline and follow-up imaging for evidence of acute ischemia/recent infarct (i.e., the acute lesion) using the validated visual scores to assess 3 features: (1) acute lesion visibility; (2) location and extent of the lesion; and (3) lesion swelling.

\section{Acute lesion visibility}

We graded acute lesion visibility on a 3-point ordinal scale to reflect the range of progressive tissue changes seen after ischemic stroke, i.e., increasingly more visible CT hypoattenuation or MRI T2-weighted hyperintensity (e.g., figure 1). This schema reflects well-described tissue changes ${ }^{3,4,16,17}$ :

- $\quad$ On CT, we defined acute lesion visibility as grade 0 (no acute lesion visible); subtle, or grade 1 (gray matter attenuation equal to that of normal white matter); and severe, or grade 2 (gray and/or white matter attenuation lower than normal white matter).

Figure $1 \mathrm{CT}$ and MRI examples of grading of acute ischemic lesion visibility

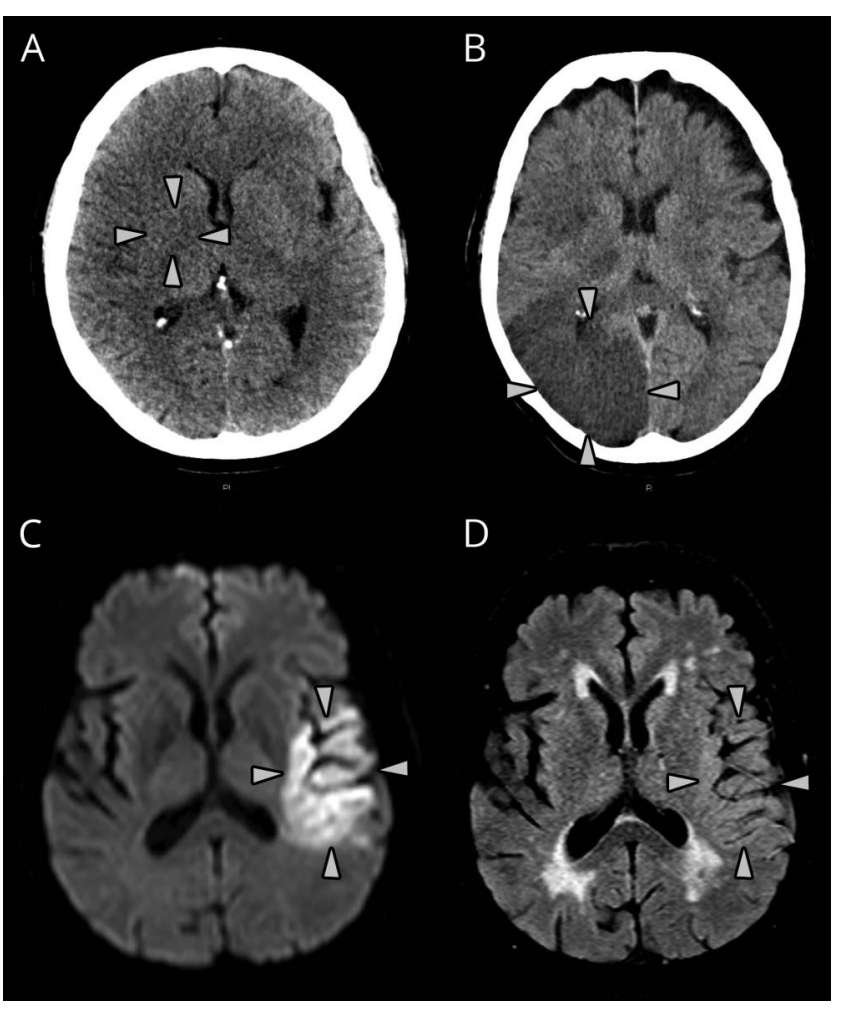

(A) CT lesion visibility grade 1; attenuation of affected gray matter (right lentiform nucleus, arrows) equivalent to normal surrounding white matter. (B) CT lesion visibility grade 2; attenuation of affected right occipital lobe (gray and white matter, arrows) less than normal white matter. (C) Diffusionweighted and (D) fluid-attenuated inversion recovery (T2-based) imaging demonstrating MRI lesion visibility grade 1; acute lesion is clearly visible in panel C (arrows) but only faintly visible in panel D (arrows).
- $\quad$ On MRI, we defined lesions as grade 0 (no lesion); subtle, or grade 1 (hyperintense area on DWI but not on other T2-weighted sequences); and severe or grade 2 (hyperintense on T2-weighted sequences with or without DWI hyperintensity). ${ }^{4}$

\section{Location and extent of acute lesion}

We used the IST-3 Ischemic Lesion Score and ASPECTS (Alberta Stroke Program Early CT Score) to assess the location and extent of acute lesions. ${ }^{18,19}$

The IST-3 Ischemic Lesion Score records the lesion extent according to common patterns of infarction in all major vascular territories (anterior, middle $[\mathrm{MCA}]$, and posterior cerebral arteries), the brainstem, or cerebellum, each with subdivisions. It includes an 8-point scale for the MCA territory., ${ }^{4,18}$ We condensed the detailed scores into fewer groups for analysis, as described previously ${ }^{4,12}$ : $0=$ no acute lesion; $1=$ small (e.g., lacunar or small cortical lesion); 2 = medium (e.g., striatocapsular lesion or superficial MCA territory); 3 = large (e.g., complete MCA territory); and 4 = very large (e.g., complete MCA plus anterior cerebral artery territories). See data available from Edinburgh DataShare (figure e-1, dx.doi.org/10.7488/ds/2367).

ASPECTS assesses lesion extent only in the MCA territory, in 10 sections, scoring a point for each area that is affected, and ranges from $10=$ normal (no MCA territory lesion) to $0=$ acute lesion affecting the entire MCA territory. ${ }^{19}$

\section{Acute lesion swelling}

We graded tissue swelling on a validated 7-point scale based on sulcal or ventricular effacement or midline shift, from none (0) to severe (6 = midline shift with effacement of the basal cisterns). ${ }^{18}$ See data available from Edinburgh DataShare (figure e-2, dx.doi.org/10.7488/ds/2367).

We also assessed baseline scans for leukoaraiosis, atrophy, and old stroke lesions (i.e., prestroke signs) using validated scores. ${ }^{4,20-22}$ We noted the presence of any hemorrhage at follow-up.

\section{Data analysis}

We compared baseline and follow-up imaging to detect change in any of the acute ischemic lesion appearances. We subtracted the baseline imaging scores from the follow-up imaging scores. For the variables lesion visibility grade, IST-3 Ischemic Lesion Score, and swelling, a positive value represented imaging progression. For ASPECTS, a negative value represented imaging progression.

We used univariate tests to compare the alteplase and control groups and to assess for associations with imaging progression. We then used multivariable ordinal regression to identify predictors of imaging progression (in those with a significant univariate association, i.e., change in lesion visibility grade) and 6-month functional outcome. Finally, we tested for interactions with alteplase between subgroups of variables predictive of 
imaging progression $(p<0.1)$ on ordinal regression analysis. Because of the risk of confounding, we did not include ASPECTS and IST-3 Ischemic Lesion Score in the same multivariable models. We adjusted regression analyses for the key outcome predictors of age, NIHSS, time between baseline and follow-up scans, and presence of hemorrhage at follow-up, plus leukoaraiosis, atrophy, and old stroke lesions since we showed previously in IST-3 and other datasets that these prestroke signs affect lesion visibility ${ }^{16,17}$ and are adverse prognostic markers ${ }^{4,12}$ (therefore, we did not repeat these tests in the present analysis). To stabilize regression estimates, we grouped the variable time between scans into 6 time windows (0-11, 12-23, 24-35, 36-47, 48-59, 60+ hours) and we combined the 3 most severe grades of the Oxford Handicap Scale into a single category, resulting in 5 functional outcome grades $(0,1,2,3,4-6)$.

We used IBM SPSS Statistics software, version 21.0 (IBM Corp., Armonk, NY) for all analyses unless otherwise stated, and considered $p<0.05$ significant.

\section{Meta-analysis}

We used the 2014 Cochrane systematic review Thrombolysis for Acute Ischemic Stroke ${ }^{23}$ to identify randomized controlled trials of IV alteplase that reported outcomes by imaging assessment of ischemic lesions at baseline and follow-up. Specifically, we sought comparable data for the 3 imaging features examined in IST-3: namely, acute lesion visibility; lesion extent (scored as lesion volume if assessed at only one time point, and lesion growth if assessed at more than one time point); and lesion swelling. In addition, for each relevant trial identified, we searched PubMed for any post hoc or subgroup analyses published up to the end of January 2018.

We used Comprehensive Meta-Analysis software, version 2 (Biostat, Englewood, NJ) to compute odds ratios (ORs) of the alteplase effect for each trial dataset and to calculate summary statistics using a random effects model. We used $I^{2}$ statistics to assess heterogeneity between studies.

\section{Data availability}

IST-3 data are available on request to bona fide researchers via Edinburgh DataShare (datashare.is.ed.ac.uk/handle/10283/ 1931).

\section{Classification of evidence}

Our primary objective was to determine whether alteplase alters the development of ischemic lesions on brain imaging after stroke. This study provides Class II evidence that IV alteplase $(0.9 \mathrm{mg} / \mathrm{kg})$ impedes the development of ischemic brain lesions according to 2 distinct imaging features. In IST3 , alteplase reduced short-term progression in lesion visibility (adjusted OR $=0.77,95 \%$ confidence interval $[\mathrm{CI}]$ 0.67-0.89), and when combined in meta-analysis with all available randomized controlled trial data, alteplase reduced the extent of the ischemic brain lesion on follow-up imaging (adjusted OR $=0.85$, 95\% CI 0.76-0.95).

\section{Results}

IST-3 recruited 3,035 patients. Baseline or follow-up imaging was not available for central review in $18(0.6 \%)$ and 105 patients $(3.5 \%)$, respectively. Central imaging review did not occur if the patient had died or was too unwell at follow-up, or if completed scans were never received centrally or were corrupted. Thus, expert-reviewed baseline and follow-up imaging was available for 2,916 patients (96.1\%) (data available from Edinburgh DataShare, figure e-3, dx.doi.org/10.7488/ ds/2367). Most had noncontrast CT performed at baseline $(2,861,98.1 \%)$ and at follow-up $(2,766,94.9 \%)$. MRI was used in 55 and 150 patients, respectively.

For the 2,916 patients included in this analysis, 1,416 (48.6\%) were male, median age was 81 years (interquartile range [IQR] 72-86 years), and the median baseline NIHSS score was 11 (IQR 6-17). Median time from stroke onset to baseline scan was 154 minutes (IQR 105-215 minutes) while the median time between baseline and follow-up scans was 26 hours (IQR 24-36 hours). Treatment allocation was 1,474 patients $(50.5 \%)$ to alteplase and $1,442(49.5 \%)$ to control. We found no differences in demographic or clinical characteristics between the 2,916 patients with complete imaging data in these analyses and the 3,035 patients in the whole IST3 trial (data not shown).

Among the 2,916 patients with complete imaging data, baseline demographic, clinical, and imaging variables were not different between treatment groups (table 1), except that patients in the control group had a slightly longer time lapse between baseline and follow-up imaging (IQR 24-40 vs 23-30 hours in the group allocated to alteplase, $p<0.001)$.

\section{Change in acute lesion between baseline and follow-up in IST-3}

We identified an acute lesion (any of reduced tissue CT attenuation/increased T2-weighted hyperintensity, or swelling) in 1,183 patients at baseline and 2,124 patients at follow-up. Most acute lesions involved the MCA territory (baseline 1,088/ $1,178,92.4 \%$, follow-up $1,722 / 2,119,81.3 \%)$. We identified any hemorrhage on $479 / 2,916$ (16.4\%) follow-up scans (table 2).

Lesion visibility grade changed between baseline and follow-up in $1,994 / 2,916$ patients $(68.4 \%)$. Most patients had no visible lesion at baseline $(1,754 / 2,916,60.2 \%)$; in contrast, $2,119 /$ $2,916(72.7 \%)$ had a visible lesion at follow-up (table 2$)$. The prevalence of visible ischemic lesions at baseline did not differ between patients who presented early (0-3 hours from symptom onset $[40.4 \%])$ vs later $(4-6$ hours $[40.8 \%])\left(\chi^{2}=0.04, p=\right.$ $0.834)$. Some lesions completely disappeared $(76 / 2,916,2.6 \%)$ between baseline and follow-up, fewer became less visible but remained present $(11 / 2,916,0.4 \%)$. Overall, lesion visibility increased in fewer patients allocated to alteplase $(936 / 1,474$, $63.5 \%)$ than to control $(971 / 1,442,67.3 \%)$, and lesion visibility decreased in more patients allocated to alteplase $(46 / 1,474$, $3.1 \%)$ than to control $(41 / 1,442,2.8 \%)(p=0.007)$ (table 3$)$. 
Table 1 Demographic, clinical, and baseline imaging characteristics of patients allocated to alteplase vs those allocated to control $(n=2,916)$

\begin{tabular}{|c|c|c|c|}
\hline \multirow[b]{2}{*}{ Characteristic } & \multicolumn{2}{|l|}{ Treatment allocation } & \multirow[b]{2}{*}{$p$ Value for difference } \\
\hline & Alteplase $(n=1,474)$ & Control $(n=1,442)$ & \\
\hline Age, y & $81(72-86)$ & $81(71-86)$ & 0.712 \\
\hline Sex, male & $720(48.8)$ & $696(48.3)$ & 0.754 \\
\hline NIHSS score & $11(6-18)$ & $11(6-17)$ & 0.953 \\
\hline Time from stroke onset to baseline scan, $\min$ & $155(106-214)$ & $151(105-216)$ & 0.966 \\
\hline Time from baseline to follow-up scan, $h$ & $26(23-30)$ & $26(24-40)$ & $<0.001$ \\
\hline OHS grade at 6 mo & $3(2-6)$ & $4(2-6)$ & 0.152 \\
\hline \multicolumn{4}{|l|}{ Baseline imaging appearances } \\
\hline Visible CT hypoattenuation or T2-weighted MRI hyperintensity of brain & $603(40.9)$ & $580(40.2)$ & 0.706 \\
\hline Brain swelling & $348(23.6)$ & $327(22.7)$ & 0.551 \\
\hline ASPECTS & $10(8-10)$ & $10(8-10)$ & 0.388 \\
\hline IST-3 ischemic lesion score & $0(0-2)$ & $0(0-2)$ & 0.542 \\
\hline Leukoaraiosis & $744(50.5)$ & $745(51.7)$ & 0.737 \\
\hline Atrophy & $1,131(76.7)$ & $1,114(77.3)$ & 0.521 \\
\hline Old stroke lesion & $676(45.9)$ & $628(43.6)$ & 0.210 \\
\hline
\end{tabular}

Abbreviations: ASPECTS = Alberta Stroke Program Early CT Score; IST-3 = Third International Stroke Trial; NIHSS = NIH Stroke Scale; OHS = Oxford Handicap Scale.

Results are median (interquartile range) or $\mathrm{n}(\%)$ as appropriate.

Acute lesion extent increased in 1,344/2,916 (46.1\%) on the IST-3 Ischemic Lesion Score and 1,241/2,916 (42.6\%) on ASPECTS among all patients. Although there were slightly fewer patients with lesion growth and more with a reduction in acute lesion extent between baseline and follow-up (on either IST-3 Ischemic Lesion Score or ASPECTS) among those allocated to alteplase vs those allocated to control, these changes were not significant in IST-3 (tables 2 and 3).

Swelling changed (mostly increased) between baseline and follow-up in 1,494/2,914 patients (51.3\%) overall (table 2). However, there was no difference in the change in swelling from baseline to follow-up scanning between treatment groups (table 3).

On ordinal regression analysis, compared with control, patients allocated to alteplase were less likely to show an increase in acute lesion visibility grade between baseline and follow-up scans ( $\mathrm{OR}=0.77,95 \%$ CI $0.67-0.89, p<0.001$ ) (table 4). Leukoaraiosis on baseline imaging independently predicted a less visible lesion on follow-up imaging $(\mathrm{OR}=$ 0.78 , 95\% CI $0.67-0.91, p=0.002$ ), but patients with leukoaraiosis were less likely to have a visible lesion both at baseline $(562 / 1,489,37.7 \%$ with leukoaraiosis vs $621 / 1,427$, $43.5 \%$ without leukoaraiosis, $\left.\chi^{2}=10.1, p=0.002\right)$ and followup $(1,034 / 1,489,69.4 \%$ with leukoaraiosis vs $1,090 / 1,427$,
$76.4 \%$ without, $\left.\chi^{2}=17.7, p<0.001\right)$. The following independently predicted development of a more visible lesion at follow-up: higher NIHSS score at randomization $(\mathrm{OR}=1.06$, 95\% CI 1.05-1.07, $p<0.001)$; the presence of an old stroke lesion at baseline ( $\mathrm{OR}=1.19,95 \% \mathrm{CI} 1.03-1.37, p=0.017)$; or hemorrhage on follow-up imaging $(\mathrm{OR}=1.62,95 \% \mathrm{CI}$ $1.34-1.96, p<0.001)$. Our results did not change if patients with MRI (at either baseline or follow-up) were excluded: in those with $\mathrm{CT}$ at both time points, OR for the effect of treatment on change in lesion visibility grade $=0.76$ (95\% CI $0.66-0.88, p<0.001, \mathrm{n}=2,731$; full data not shown).

We found no evidence of an interaction between alteplase and prespecified subgroups on change in acute lesion visibility grade between baseline and follow-up (ordinal regression analysis): baseline stroke severity mild (NIHSS score $<8$ ) vs moderatesevere (NIHSS score $\geq 8)(p=0.842)$; presence vs absence of leukoaraiosis, atrophy, or old stroke lesions $(p=0.871, p=$ $0.358, p=0.239$, respectively) (data available from Edinburgh DataShare, figure e-4, dx.doi.org/10.7488/ds/2367).

\section{Effect of imaging appearances and clinical features on 6-month outcome in IST-3}

On univariate analysis, the change in IST-3 Ischemic Lesion Score $(r=0.21, p<0.001)$, ASPECTS $(r=-0.35, p<0.001)$, acute lesion visibility grade $(r=0.22, p<0.001)$, and swelling 
Table 2 Results of central expert panel assessment for the imaging variables assessed at baseline and follow-up $(n=2,916)$

\begin{tabular}{|c|c|c|c|}
\hline Imaging variable & Options & $\begin{array}{l}\text { Baseline scan } \\
(2,861 \mathrm{CT}, 55 \\
\text { MRI) }\end{array}$ & $\begin{array}{l}\text { Follow-up scan } \\
(2,766 \mathrm{CT}, 150 \mathrm{MRI})\end{array}$ \\
\hline $\begin{array}{l}\text { Presence of an acute lesion (either change in tissue CT } \\
\text { attenuation/T2-weighted intensity or swelling) }\end{array}$ & & $1,183(40.6)$ & $2,124(72.8)$ \\
\hline \multirow[t]{3}{*}{ Visibility grade } & $0=$ No attenuation $/$ intensity change & $1,754(60.2)$ & $797(27.3)$ \\
\hline & 1 = Early tissue changes & $974(33.4)$ & $204(7.0)$ \\
\hline & $2=$ Late tissue changes & $188(6.4)$ & $1,915(65.7)$ \\
\hline \multirow[t]{6}{*}{ Location of acute lesion } & ACA territory & $4 / 1,178(0.3)$ & $37 / 2,119(1.7)$ \\
\hline & MCA territory & $1,088(92.4)$ & $1,722(81.3)$ \\
\hline & PCA territory & $39(3.3)$ & $108(5.1)$ \\
\hline & Vertebrobasilar territory & $18(1.5)$ & $59(2.8)$ \\
\hline & Borderzone & $7(0.6)$ & $14(0.7)$ \\
\hline & Multiple arterial territories & $22(1.9)$ & $179(8.4)$ \\
\hline \multirow[t]{11}{*}{ ASPECTS } & 10 & $1,825(62.6)$ & $1,118(38.3)$ \\
\hline & 9 & $174(6.0)$ & $232(8.0)$ \\
\hline & 8 & $205(7.0)$ & $230(7.9)$ \\
\hline & 7 & $180(6.2)$ & $265(9.1)$ \\
\hline & 6 & $142(4.9)$ & $198(6.8)$ \\
\hline & 5 & $98(3.4)$ & $176(6.0)$ \\
\hline & 4 & $77(2.6)$ & $116(4.0)$ \\
\hline & 3 & $78(2.7)$ & $132(4.5)$ \\
\hline & 2 & $70(2.4)$ & $112(3.8)$ \\
\hline & 1 & $32(1.1)$ & $134(4.6)$ \\
\hline & 0 & $35(1.2)$ & $203(7.0)$ \\
\hline \multirow[t]{5}{*}{ IST-3 ischemic lesion score } & $0=$ No acute lesion & $1,738(59.6)$ & $797(27.3)$ \\
\hline & $1=$ Small & $194(6.7)$ & $432(14.8)$ \\
\hline & 2 = Medium & $485(16.6)$ & $723(24.8)$ \\
\hline & $3=$ Large & $254(8.7)$ & $414(14.2)$ \\
\hline & 4 = Very large & $245(8.4)$ & $550(18.9)$ \\
\hline \multirow[t]{7}{*}{ Degree of tissue swelling } & None & $2,241(76.9)$ & $1,244 / 2,914(42.7)$ \\
\hline & Effacement of sulci only & $534(18.3)$ & $735(25.2)$ \\
\hline & Effacement of sulci and lateral ventricle (minor) & $140(4.8)$ & $658(22.6)$ \\
\hline & $\begin{array}{l}\text { Effacement of sulci and lateral ventricle } \\
\text { (complete) }\end{array}$ & 0 & $55(1.9)$ \\
\hline & Effacement of sulci, lateral and third ventricles & 0 & $15(0.5)$ \\
\hline & $\begin{array}{l}\text { Effacement of sulci, lateral and third ventricles } \\
\text { plus midline shift }\end{array}$ & $1(0.03)$ & $124(4.3)$ \\
\hline & $\begin{array}{l}\text { Effacement sulci, lateral and third ventricles, } \\
\text { midline shift plus effacement of basal cisterns }\end{array}$ & 0 & $83(2.8)$ \\
\hline Any hemorrhage on follov & & - & $479(16.4)$ \\
\hline
\end{tabular}

Abbreviations: ACA = anterior cerebral artery; ASPECTS = Alberta Stroke Program Early CT Score; IST-3 = Third International Stroke Trial; MCA = middle cerebral artery; PCA = posterior cerebral artery.

Results are $n(\%)$. Data for location of acute lesion and degree of tissue swelling were not available for all patients at both time points. Presence of hemorrhage was assessed only at follow-up. 
Table 3 Univariate analyses comparing baseline to follow-up imaging progression between the alteplase vs control groups

\begin{tabular}{|c|c|c|c|c|}
\hline Imaging variable & $\begin{array}{l}\text { Imaging change between } \\
\text { scans }^{a}\end{array}$ & $\begin{array}{l}\text { Alteplase } \\
\text { group }\end{array}$ & $\begin{array}{l}\text { Control } \\
\text { group }\end{array}$ & $\begin{array}{l}p \text { Value for difference } \\
\text { between alteplase and control } \\
\text { groups }{ }^{\mathbf{b}}\end{array}$ \\
\hline \multirow[t]{3}{*}{ Lesion visibility grade $(n=2,916)$} & Less visible & $46(3.1)$ & $41(2.8)$ & 0.007 \\
\hline & No change & $492(33.4)$ & $430(29.8)$ & \\
\hline & More visible & $936(63.5)$ & $971(67.3)$ & \\
\hline \multirow{3}{*}{$\begin{array}{l}\text { IST-3 ischemic lesion score }(n= \\
2,916)\end{array}$} & Smaller lesion & $112(7.6)$ & $101(7.0)$ & 0.149 \\
\hline & No change & $695(47.2)$ & $649(45.0)$ & \\
\hline & Larger lesion & $667(45.3)$ & $692(48.0)$ & \\
\hline \multirow[t]{3}{*}{ ASPECTS $(n=2,916)$} & Smaller lesion & $141(9.6)$ & $134(9.3)$ & 0.768 \\
\hline & No change & $639(43.4)$ & $602(41.7)$ & \\
\hline & Larger lesion & $694(47.1)$ & $706(49.0)$ & \\
\hline \multirow[t]{3}{*}{ Swelling $(n=2,914)$} & Less swelling & $35(2.4)$ & $45(3.1)$ & 0.402 \\
\hline & No change & $727(49.3)$ & $693(48.1)$ & \\
\hline & More swelling & $712(48.3)$ & $702(48.8)$ & \\
\hline
\end{tabular}

Abbreviations: ASPECTS = Alberta Stroke Program Early CT Score; IST-3 = Third International Stroke Trial.

Results represent $\mathrm{n}(\%)$ within the alteplase and control groups.

a Scalar data have been condensed for simplification in this table.

${ }^{b}$ The $p$ values are derived from noncondensed data.

( $r=0.40, p<0.001)$ between baseline and follow-up imaging were all associated with 6-month functional outcome such that a worsening of imaging appearances between baseline and 24-48 hours was associated with worse 6-month functional outcome.

On ordinal regression analysis, the following were independent predictors of poor outcome: greater age $(\mathrm{OR}=$ $0.97, p<0.001)$; higher baseline NIHSS score $(\mathrm{OR}=0.86, p<$ $0.001)$; leukoaraiosis ( $\mathrm{OR}=0.72, p<0.001)$ or atrophy $(\mathrm{OR}=0.77, p=0.013)$ on baseline imaging; increasing acute lesion extent $(\mathrm{OR}=0.92, p<0.001)$ or swelling $(\mathrm{OR}=0.73$, $p<0.001)$ between baseline and follow-up imaging; and hemorrhage on follow-up imaging $(\mathrm{OR}=0.74, p=0.017)$. Treatment with alteplase $(\mathrm{OR}=1.36, p<0.001)$ and increased time between baseline and follow-up imaging ( $\mathrm{OR}=$ $1.10, p=0.009)$ independently predicted better outcome at 6 months. Old stroke lesions and increasing visibility grade of the acute lesion between baseline and follow-up did not independently predict outcome (table 5). Results were similar if IST-3 Ischemic Lesion Score was used instead of ASPECTS to assess change in lesion extent (data not shown).

Table 4 Ordinal regression analysis of associations between change in lesion visibility grade between baseline and 24- to 48-hour follow-up (dependent variable) and potential clinical and imaging predictors

\begin{tabular}{|c|c|c|c|c|}
\hline Change in lesion visibility grade $(n=2,916)$ & Source data & Odds ratio & $95 \% \mathrm{Cl}$ & $p$ Value \\
\hline Increasing age, y & $81(72-86)$ & 1.01 & $1.00-1.01$ & 0.100 \\
\hline Increasing baseline NIHSS score & $11(6-17)$ & 1.06 & $1.05-1.07$ & $<0.001$ \\
\hline Leukoaraiosis on baseline imaging & $1,489(51.1)$ & 0.78 & $0.67-0.91$ & 0.002 \\
\hline Atrophy on baseline imaging & $2,245(77.0)$ & 0.84 & $0.69-1.02$ & 0.073 \\
\hline Old stroke lesion(s) on baseline imaging & $1,304(44.7)$ & 1.19 & $1.03-1.37$ & 0.017 \\
\hline Increasing time from baseline to follow-up imaging (12-h groups) & $26(24-36)$ & 1.05 & $0.99-1.12$ & 0.111 \\
\hline Treatment with alteplase & $1,474(50.5)$ & 0.77 & $0.67-0.89$ & $<0.001$ \\
\hline Any hemorrhage on follow-up imaging & $479(16.4)$ & 1.62 & $1.34-1.96$ & $<0.001$ \\
\hline
\end{tabular}

Abbreviations: $\mathrm{Cl}=$ confidence interval; NIHSS = NIH Stroke Scale.

Source data summary provided as median (interquartile range) or $\mathrm{n}(\%)$. Odds ratio $>1$ indicates a more visible lesion. 


\section{Meta-analysis}

There have been no new randomized controlled trials of IV alteplase vs control since publication of the Cochrane review in 2014. The Cochrane review already contained a metaanalysis assessing the alteplase effect on symptomatic edema in 6 trials (including IST-3), which we did not repeat. Two of the trial datasets identified using Cochrane (ECASS [European Cooperative Acute Stroke Study], EPITHET [Echoplanar Imaging Thrombolytic Evaluation Trial] $)^{24,25}$ had examined lesion growth between baseline and follow-up similar to IST-3, while 3 of the trials (NINDS [National Institute of Neurological Disorders and Stroke], ATLANTIS [Alteplase Thrombolysis for Acute Noninterventional Therapy in Ischemic Stroke] A \& B $)^{26-28}$ had measured lesion volume on follow-up imaging alone (total $\mathrm{n}=1,841$ ).

On meta-analysis, we found that alteplase impeded lesion growth $(\mathrm{OR}=0.87,95 \% \mathrm{CI} 0.76-0.99)$ and was associated with smaller lesion volumes on follow-up imaging $(\mathrm{OR}=0.82,95 \% \mathrm{CI}$ $0.67-1.00)$. When combined in a single meta-analysis including nearly 5,000 patients from 6 trials (including IST-3), alteplase reduced progression in the extent of the ischemic lesion (increased lesion growth or greater lesion volume on follow-up imaging $(\mathrm{OR}=0.85,95 \% \mathrm{CI} 0.76-0.95, p=0.004)$ (figure 2).

\section{Discussion}

In this large randomized trial with baseline and follow-up CT or MRI available for blinded central review in more than $97 \%$ of patients, we found that during the first 24 to 48 hours after the onset of ischemic stroke, patients allocated to alteplase were less likely to show progression in the visibility of the acute lesion compared with controls. Remarkably, less than $50 \%$ of patients in both treatment arms showed any lesion growth. IV alteplase did not reduce significantly the extent of the acute lesion or alter the degree of lesion swelling in IST-3, although there were fewer patients with larger lesions and more patients with smaller or stable lesions at 24 to 48 hours who received alteplase than control. It seems likely that IST-3 was underpowered to detect this subtle change in lesion extent, but when IST-3 data were combined in a meta-analysis of 6 randomized controlled trials of alteplase including nearly 5,000 patients, we found that alteplase was associated with a significant reduction in progression of the ischemic lesion extent. This alteplase effect includes a reduction in lesion growth between baseline and follow-up and a smaller lesion volume at follow-up. We postulate that IV alteplase resulted in fewer patients either converting an area of reversible ischemia to infarction, or expanding their ischemic lesion into tissue that was unaffected at baseline-both effects result in less injured brain tissue and may explain the better functional outcome seen following treatment with alteplase.

Background prestroke brain imaging features and baseline stroke severity were also independent predictors of change in acute lesion visibility from baseline to follow-up in IST-3. Patients with old infarcts were more likely to have increased acute lesion visibility at 24 to 48 hours. Perhaps individuals with prior infarcts are more likely to develop tissue injury if they experience another stroke than are patients without old infarcts on imaging, as suggested for lesion visibility on MRI in patients with minor stroke. ${ }^{29}$ Harder to explain is the finding that prestroke leukoaraiosis appeared to reduce progression of

Table 5 Ordinal regression analysis of 6-month functional outcome (dependent variable) and potential predictors: Clinical and imaging characteristics (change in the acute lesion from baseline to 24- to 48-hour follow-up; prestroke features)

\begin{tabular}{|c|c|c|c|c|}
\hline OHS grade at $6 \mathrm{mo}(n=2,916)$ & Source data & Odds ratio & $95 \% \mathrm{Cl}$ & $p$ Value \\
\hline Increasing age, y & $81(72-86)$ & 0.97 & $0.96-0.98$ & $<0.001$ \\
\hline Increasing baseline NIHSS score & $11(6-17)$ & 0.86 & $0.85-0.87$ & $<0.001$ \\
\hline Leukoaraiosis on baseline imaging & $1,488(51.1)$ & 0.72 & $0.60-0.85$ & $<0.001$ \\
\hline Atrophy on baseline imaging & $2,243(77.0)$ & 0.77 & $0.62-0.95$ & 0.013 \\
\hline Old stroke lesion(s) on baseline imaging & $1,304(44.7)$ & 0.92 & $0.78-1.07$ & 0.277 \\
\hline Increased lesion visibility grade from baseline to follow-up imaging & $1,906(65.4)$ & 0.97 & $0.88-1.07$ & 0.525 \\
\hline Worsening ASPECTS (more extensive lesion) from baseline to follow-up imaging & $1,399(48.0)$ & 0.92 & $0.88-0.96$ & $<0.001$ \\
\hline Increased extent of tissue swelling from baseline to follow-up imaging & $1,414(48.5)$ & 0.73 & $0.66-0.79$ & $<0.001$ \\
\hline Increasing time from baseline to follow-up imaging (12-h groups) & $26(24-36)$ & 1.10 & $1.02-1.18$ & 0.009 \\
\hline Treatment with alteplase & $1,474(50.6)$ & 1.36 & $1.17-1.58$ & $<0.001$ \\
\hline Any hemorrhage on follow-up imaging & $479(16.4)$ & 0.74 & $0.57-0.95$ & 0.017 \\
\hline
\end{tabular}

Abbreviations: ASPECTS = Alberta Stroke Program Early CT Score; CI = confidence interval; NIHSS = NIH Stroke Scale; OHS = Oxford Handicap Scale. Source data provided as median (interquartile range) or $\mathrm{n}(\%)$. Odds ratio $>1$ indicates an increased likelihood of a better outcome. 
Figure 2 Meta-analysis of data from randomized controlled trials of alteplase that assessed short-term lesion growth or lesion extent on follow-up imaging

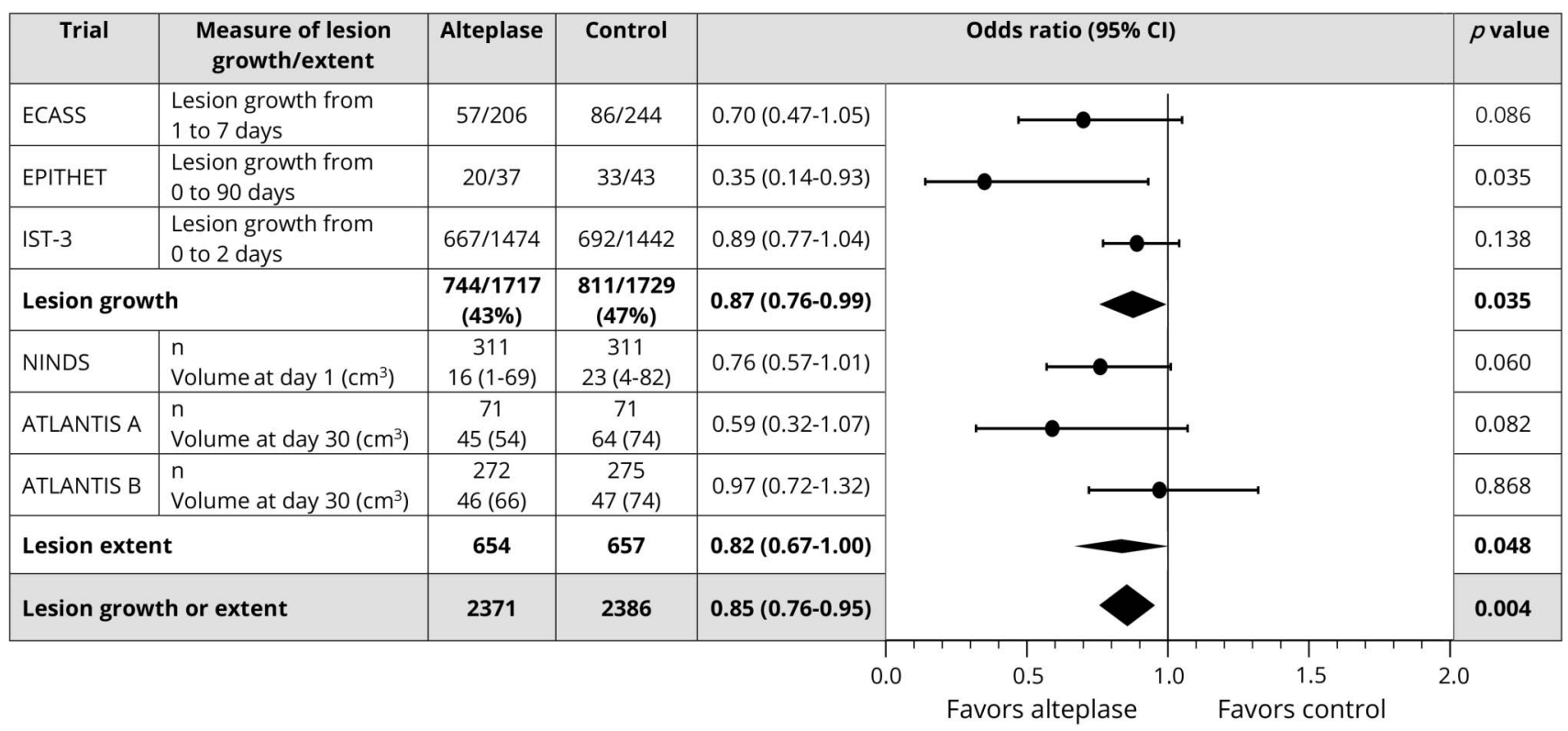

For all studies in the combined analysis, $I^{2}=31.5 \%$. ATLANTIS = Alteplase Thrombolysis for Acute Noninterventional Therapy in Ischemic Stroke; $\mathrm{Cl}=$ confidence interval; ECASS = European Cooperative Acute Stroke Study; EPITHET = Echoplanar Imaging Thrombolytic Evaluation Trial; IST-3 = Third International Stroke Trial; NINDS = National Institute of Neurological Disorders and Stroke.

acute lesion visibility at follow-up. This may be because acute lesions were less often seen in patients with chronically abnormal white matter at baseline, a discrepancy that increased on follow-up imaging, but the reason for this is unclear.

Increased stroke severity (NIHSS) at baseline was a powerful independent predictor for increased lesion visibility on followup imaging in IST-3 in keeping with current and previously demonstrated associations between NIHSS, other imaging measures of severity (hyperattenuated arteries, arterial occlusion on angiography, acute lesion extent), and outcome after ischemic stroke. ${ }^{10,11,30}$ However, we found no evidence of an interaction between alteplase and severe vs mild stroke or presence/absence of prestroke features on change in acute lesion visibility; i.e., alteplase worked equally well across all of these subgroups.

Worsening of imaging appearances from baseline to 24-48 hours independently predicted poor outcome at 6 months in IST-3. Specifically, greater change in acute lesion extent on ASPECTS and increased tissue swelling were both associated with poor outcome. In addition, increased time from baseline to follow-up imaging was associated with better functional outcome, perhaps because of residual confounding with milder strokes being reimaged later. However, despite a significant univariate correlation between greater lesion visibility from baseline to 24- to 48-hour follow-up imaging and functional outcome, changes in lesion visibility did not independently predict outcome. This may reflect that increased lesion visibility in patients at follow-up in our analysis may include new infarcts appearing since baseline imaging (likely to affect functional outcome) and early infarcts at baseline becoming more visible secondary to the expected short-term tissue changes of infarct (less likely to affect functional outcome). Whether the transition from ischemic to infarcted brain can be reliably differentiated using unenhanced $\mathrm{CT}$ or basic MRI sequences remains unproven.

Lack of an association between alteplase and change in the degree of swelling at 24- to 48-hour follow-up appears to contrast with the significant excess of symptomatic brain swelling (that is, severe swelling on follow-up imaging plus neurologic deterioration within 7 days) among alteplasetreated patients in the primary IST- 3 report. ${ }^{12}$ However, the current analysis examined all grades of swelling rather than only symptomatic swelling, and as noted above, progressive swelling was associated with worse functional outcome at 6 months.

The limitations of IST-3 have been discussed previously, ${ }^{12}$ chiefly the potential for bias because of the open trial design; however, for the present analyses, all readers were masked to clinical data and treatment allocation. In IST-3, investigators were required to perform follow-up imaging between 24 and 48 hours unless the patient deteriorated clinically, in which case immediate rescanning was required; patients who deteriorated and hence were scanned sooner were more likely to have poor 6-month outcomes. Patients treated with alteplase underwent follow-up imaging marginally sooner than controls but had better outcomes overall. It is likely that this marginal difference in scan interval may reflect the open design and the 
less-pressing need to reimage patients known to have been allocated to the control group. Although almost all patients had CT imaging at baseline and follow-up, a few patients were scanned with different modalities at the 2 time points. It may not be appropriate to compare acute lesion extent and visibility between CT and MRI, though it is perhaps reasonable to assume that classification of swelling would not differ between modalities. However, the results remained the same when those with MRI were excluded from the analysis assessing change in lesion visibility; we have therefore left all patients in for completeness. Validated quantitative computational methods for the assessment of brain imaging are not yet available for all of the characteristics we assessed in IST-3. Most important, computational lesion volume measurement does not distinguish between an increase in volume due to a true change in lesion extent and an increase in volume due to more swelling in a lesion of the same extent. We and others are developing computational lesion assessment methods, ${ }^{31}$ but their superiority over human visual rating remains unproven. Finally, angiographic imaging at baseline and followup was not available. We are aware that we do not know whether and to what extent treatment with alteplase was associated with arterial recanalization, and tissue reperfusion. The strengths of IST-3 include its large sample size, the inclusion of patients with a wide range of clinical characteristics and baseline imaging appearances, and central masked review of all imaging and visual scoring methods that had undergone extensive independent validation. ${ }^{16,17}$

We provide robust evidence that in ischemic stroke, allocation to alteplase was associated with less progression in the extent (based on the totality of the randomized evidence from the meta-analysis) and visibility (in IST-3 alone) of the acute lesion on short-term imaging follow-up. These findings may reflect less tissue damage among patients treated with alteplase and help explain how alteplase improves functional outcome after ischemic stroke. Alteplase did not alter the development of lesion swelling, but progression of lesion extent and swelling were both associated with poorer clinical outcome at 6 months. Imaging biomarkers of the effect of alteplase on ischemic brain will be valuable for clinical practice and research. Our findings indicate that early changes in the ischemic brain lesion are measurable on imaging, that these imaging appearances can predict outcome, and that treatment with alteplase limits progression of the ischemic lesion, which may in turn act as a surrogate for an improved functional outcome.

\section{Author contributions}

Dr. Mair contributed to the design of this work, analyzed and interpreted the data, and drafted the manuscript. Prof. von Kummer contributed to the design of this work, to data collection and interpretation, and critically revised the manuscript. Dr. Morris contributed to data collection and critically revised the manuscript. Dr. von Heijne contributed to data collection and critically revised the manuscript. Dr. Bradey contributed to data collection and critically revised the manuscript. Prof. Cala contributed to data collection and critically revised the manuscript. Dr. Peeters contributed to data collection and critically revised the manuscript. Prof. Farrall contributed to data collection and critically revised the manuscript. Dr. Adami contributed to data collection and critically revised the manuscript. Dr. Potter contributed to data collection and critically revised the manuscript. Prof. Sandercock contributed to the design of this work, to data collection and interpretation, and critically revised the manuscript. Prof. Lindley contributed to the design of this work, to data interpretation, and critically revised the manuscript. Prof. Wardlaw helped design IST-3, designed and supervised the IST-3 imaging data collection, coordinated the image reading panel, managed all IST-3 imaging analysis, data processing and supervised the statistical analysis, and drafted and critically revised the manuscript.

\section{Study funding}

IST-3 was funded from a large number of sources (data available from Edinburgh DataShare, eAppendix, dx.doi.org/ $10.7488 / \mathrm{ds} / 2367$ ) but chiefly the UK Medical Research Council (MRC G0400069 and EME 09-800-15) and the UK Stroke Association.

\section{Disclosure}

G. Mair reports no disclosures relevant to the manuscript. R. von Kummer is editor-in-chief of Neuroradiology, served on the data monitoring and safety committees for Impact-24, ReSPECT ESUS, ReSPECT CVT, ECASS 4, SWIFT DIRECT and bills BrainsGate, Applied Clinical Intelligence, LLC, and Parexel for his time spent on these studies. He is adjudicating the images of SITS Open and bills Karolinska Institutet per case. Z. Morris, A. von Heijne, N. Bradey, and L. Cala report no disclosures relevant to the manuscript. A. Peeters: Boehringer Ingelheim. A. Farrall, A. Adami, and G. Potter report no disclosures relevant to the manuscript. P. Sandercock was the chief investigator of the IST-3 trial, which received a donation of drug and placebo for 300 patients in the IST-3 pilot study. Prof. Sandercock has received lecture fees (paid to his department) from Boehringer Ingelheim. R. Lindley: Boehringer Ingelheim, Covidien. J. Wardlaw received research funding from the Medical Research Council, Efficacy and Mechanisms Evaluation, Stroke Association, Health Foundation, and Chest Heart \& Stroke Scotland for this work. Prof. Wardlaw was imaging chief investigator for IST-3, which received a donation of drug and placebo for the first 300 patients in IST-3 from Boehringer Ingelheim and initial setup of the image management system from DesAcc. Prof. Wardlaw also receives funding from the EU H2020 SVDs@Target and Fondation Leducq. Go to Neurology.org/N for full disclosures.

\section{Publication history}

Received by Neurology March 12, 2018. Accepted in final form August 14, 2018.

\section{References}

1. Simard JM, Kent TA, Chen M, Tarasov KV, Gerzanich V. Brain oedema in foca ischaemia: molecular pathophysiology and theoretical implications. Lancet Neurol 2007;6:258-268. 
2. Dzialowski I, Weber J, Doerfler A, Forsting M, von Kummer R. Brain tissue water uptake after middle cerebral artery occlusion assessed with CT. J Neuroimaging 2004; $14: 42-48$.

3. Wardlaw JM, Mielke O. Early signs of brain infarction at CT: observer reliability and outcome after thrombolytic treatment—systematic review. Radiology 2005;235: 444-453.

4. Wardlaw JM, Sandercock PAG, Lindley RI, et al. Association between brain imaging signs, early and late outcomes, and response to intravenous alteplase after acute ischaemic stroke in the Third International Stroke Trial (IST-3): secondary analysis of a randomised controlled trial. Lancet Neurol 2015;14:485-496.

5. Dzialowski I, Klotz E, Goericke S, Doerfler A, Forsting M, von Kummer R. Ischemic brain tissue water content: CT monitoring during middle cerebral artery occlusion and reperfusion in rats. Radiology 2007;243:720-726.

6. von Kummer R, Dzialowski I. Imaging of cerebral ischemic edema and neuronal death. Neuroradiology 2017;59:545-553.

7. Moseley ME, Kucharczyk J, Mintorovitch J, Cohen Y, Kurhanewicz J, Derugin N. Diffusion-weighted MR imaging of acute stroke: correlation with T2-weighted and magnetic susceptibility-enhanced MR imaging in cats. AJNR Am J Neuroradiol 1990; 11:423-429.

8. Thomalla G, Cheng B, Ebinger M, et al. DWI-FLAIR mismatch for the identification of patients with acute ischaemic stroke within $4.5 \mathrm{~h}$ of symptom onset (PRE-FLAIR): a multicentre observational study. Lancet Neurol 2011;10:978-986.

9. Emberson J, Lees KR, Lyden P, et al. Effect of treatment delay, age, and stroke severity on the effects of intravenous thrombolysis with alteplase for acute ischaemic stroke: a meta-analysis of individual patient data from randomised trials. Lancet 2014;384: 1929-1935.

10. Mair G, von Kummer R, Morris Z, et al. Effect of alteplase on the CT hyperdense artery sign and outcome after ischemic stroke. Neurology 2016;86:118-125.

11. Mair G, von Kummer R, Adami A, et al. Arterial obstruction on computed tomographic or magnetic resonance angiography and response to intravenous thrombolytics in ischemic stroke. Stroke 2017;48:353-360.

12. IST-3 Collaborative Group. The benefits and harms of intravenous thrombolysis with recombinant tissue plasminogen activator within $6 \mathrm{~h}$ of acute ischaemic stroke (the Third International Stroke Trial [IST-3]): a randomised controlled trial. Lancet 2012; 379:2352-2363.

13. Sandercock P, Lindley R, Wardlaw J, et al. Third International Stroke Trial (IST-3) of thrombolysis for acute ischaemic stroke. Trials 2008;9:37.

14. Bamford J, Sandercock P, Dennis M, Burn J, Warlow C. A prospective study of acute cerebrovascular disease in the community: the Oxfordshire Community Stroke Project-1981-86. 2. Incidence, case fatality rates and overall outcome at one year of cerebral infarction, primary intracerebral and subarachnoid haemorrhage. J Neurol Neurosurg Psychiatry 1990;53:16-22.

15. Whiteley W, Lindley R, Wardlaw J, Sandercock P. Third International Stroke Trial. Int J Stroke 2006;1:172-176.

16. Wardlaw JM, Farrall AJ, Perry D, et al. Factors influencing the detection of early computed tomography signs of cerebral ischemia: an internet-based, international multiobserver study. Stroke 2007;38:1250-1256.

17. Wardlaw JM, von Kummer R, Farrall AJ, Chappell FM, Hill M, Perry D. A large webbased observer reliability study of early ischaemic signs on computed tomography.
The Acute Cerebral CT Evaluation of Stroke Study (ACCESS). PLoS One 2010;5: e15757.

18. Wardlaw JM, Sellar RJ. A simple practical classification of cerebral infarcts on CT and its interobserver reliability. AJNR Am J Neuroradiol 1994;15:1933-1939.

19. Barber PA, Demchuk AM, Zhang J, Buchan AM. Validity and reliability of a quantitative computed tomography score in predicting outcome of hyperacute stroke before thrombolytic therapy. ASPECTS Study Group. Alberta Stroke Programme Early CT Score. Lancet 2000;355:1670-1674.

20. Fazekas F, Chawluk JB, Alavi A, Hurtig HI, Zimmerman RA. MR signal abnormalities at $1.5 \mathrm{~T}$ in Alzheimer's dementia and normal aging. AJR Am J Roentgenol 1987;149: 351-356.

21. van Swieten JC, Hijdra A, Koudstaal PJ, van Gijn J. Grading white matter lesions on CT and MRI: a simple scale. J Neurol Neurosurg Psychiatry 1990;53:1080-1083.

22. Farrell C, Chappell F, Armitage PA, et al. Development and initial testing of normal reference MR images for the brain at ages 65-70 and 75-80 years. Eur Radiol 2008; 19:177-183.

23. Wardlaw JM, Murray V, Berge E, del Zoppo GJ. Thrombolysis for acute ischaemic stroke. Cochrane Database Syst Rev 2014;7:CD000213.

24. Pantano P, Caramia F, Bozzao L, Dieler C, von Kummer R. Delayed increase in infarct volume after cerebral ischemia: correlations with thrombolytic treatment and clinical outcome. Stroke 1999;30:502-507.

25. Davis SM, Donnan G, Parsons MW, et al. Effects of alteplase beyond $3 \mathrm{~h}$ after stroke in the Echoplanar Imaging Thrombolytic Evaluation Trial (EPITHET): a placebocontrolled randomised trial. Lancet Neurol 2008;7:299-309.

26. Clark WM, Albers GW, Madden KP, Hamilton S. The rtPA (alteplase) 0- to 6-hour acute stroke trial, part A (A0276g): results of a double-blind, placebo-controlled, multicenter study. Thrombolytic Therapy in Acute Ischemic Stroke Study investigators. Stroke 2000;31:811-816.

27. Clark WM, Wissman S, Albers GW, Jhamandas JH, Madden KP, Hamilton S. Recombinant tissue-type plasminogen activator (alteplase) for ischemic stroke 3 to 5 hours after symptom onset. The ATLANTIS Study: a randomized controlled trial. Alteplase Thrombolysis for Acute Noninterventional Therapy in Ischemic Stroke. JAMA 1999;282:2019-2026.

28. The National Institute of Neurological Disorders and Stroke (NINDS) rt-PA Stroke Study Group. Effect of intravenous recombinant tissue plasminogen activator on ischemic stroke lesion size measured by computed tomography. Stroke 2000;31: 2912-2919.

29. Makin SD, Doubal FN, Dennis MS, Wardlaw JM. Clinically confirmed stroke with negative diffusion-weighted imaging magnetic resonance imaging: longitudinal study of clinical outcomes, stroke recurrence, and systematic review. Stroke 2015;46: 3142-3148.

30. Schaefer PW, Pulli B, Copen WA, et al. Combining MRI with NIHSS thresholds to predict outcome in acute ischemic stroke: value for patient selection. AJNR Am J Neuroradiol 2015;36:259-264.

31. Chen L, Carlton Jones AL, Mair G, et al. Rapid automated quantification of cerebral leukoaraiosis on CT images: a multicenter validation study. Radiology 2018;288: 573-581. 


\section{Neurology}

Effect of IV alteplase on the ischemic brain lesion at 24-48 hours after ischemic stroke Grant Mair, Rüdiger von Kummer, Zoe Morris, et al.

Neurology 2018;91;e2067-e2077 Published Online before print October 26, 2018

DOI 10.1212/WNL.0000000000006575

This information is current as of October 26, 2018

\section{Updated Information \&} Services

References

Subspecialty Collections

Permissions \& Licensing

Reprints including high resolution figures, can be found at: http://n.neurology.org/content/91/22/e2067.full

This article cites 31 articles, 12 of which you can access for free at: http://n.neurology.org/content/91/22/e2067.full\#ref-list-1

This article, along with others on similar topics, appears in the following collection(s):

Clinical trials Randomized controlled (CONSORT agreement)

http://n.neurology.org/cgi/collection/clinical_trials_randomized_contro lled_consort_agreement

CT

http://n.neurology.org/cgi/collection/ct

Infarction

http://n.neurology.org/cgi/collection/infarction

MRI

http://n.neurology.org/cgi/collection/mri

Information about reproducing this article in parts (figures,tables) or in its entirety can be found online at:

http://www.neurology.org/about/about_the_journal\#permissions

Information about ordering reprints can be found online:

http://n.neurology.org/subscribers/advertise

Neurology ${ }^{\circledR}$ is the official journal of the American Academy of Neurology. Published continuously since 1951 , it is now a weekly with 48 issues per year. Copyright Copyright (C) 2018 The Author(s). Published by Wolters Kluwer Health, Inc. on behalf of the American Academy of Neurology.. All rights reserved. Print ISSN: 0028-3878. Online ISSN: 1526-632X.

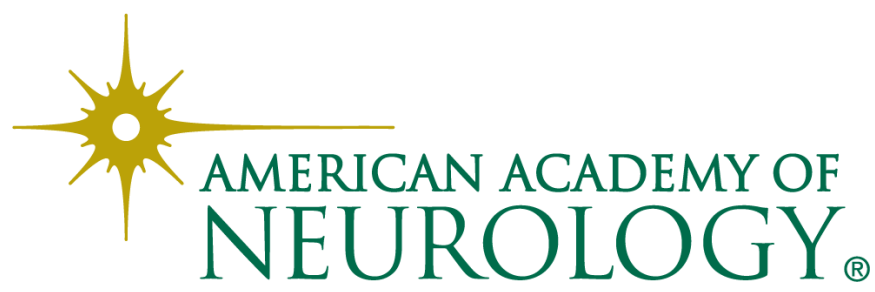

